\title{
MODELS FOR THE INTERPRETATION OF THE DIFFERENT INTERFACES Sb/InP(100) USING QUANTITATIVE RESULTS OF AES AND EELS
}

\author{
B. Gruzza, A. Porte, L. Bideux \\ Laboratoire de Physique des Milieux Condensés, CNRS 796 \\ Université Blaise Pascal - Clermond Ferrand II \\ 63177 Aubiere, Cedex, France \\ C. Jardin AND J. Miloua \\ Laboratoire de Mineralogie-Cristallographie, CNRS 805 \\ Universite Claude Bernard-Lyon I, Bd Du 11 Novembre 1918 \\ 69622 Villeurbanne, Cedex, France \\ (Received May 21, 1991; in revised form August 15, 1991)
}

\begin{abstract}
The model of quantitative interpretation of Auger electron spectroscopy (AES) results is described and some complementary electron energy loss spectroscopy (EELS) results are also reported. It is shown that the $\operatorname{In} P(100)$ surfaces perturbed by the $\mathrm{Ar}^{+}$cleaning treatment can be ordered by $\mathrm{Sb}$ deposition. The variations of different Auger lines are interpreted, and the transformations $3 \mathrm{D} \rightarrow 2 \mathrm{D}$ of the initially formed In clusters can be well-followed during the first stages of the deposition.

PACS numbers: 68 .35.Bs, 68.35.Fx, 72.80.Ey, 82.80.Pv
\end{abstract}

\section{Introduction}

The InP(100) samples are cleaned, in a UHV chamber, by low-energy $\mathrm{Ar}^{+}$ ion bombardment $\left(300 \mathrm{eV}, 2 \mu \mathrm{A} / \mathrm{cm}^{2}\right)$ [1]. It is observed then that, even if the contamination layer is easily removed, the surfaces are generally depleted from phosphorus and indium crystallites are created [2]. The structure of these microscopic crystallites was determined by the authors [3] using a quantitative interpretation of the Auger electron spectroscopy (AES), the electron energy loss spectroscopy (EELS), and the elastic peak electron spectroscopy (EPES) results.

By condensation of one of the elements from Group V, such as antimony, on the $\operatorname{InP}(100)$ surface at room temperature, all the indium crystallites form with the deposit an InSb overlayer [4]. In order to establish the mechanism of the formation of $\mathrm{Sb} / \mathrm{InP}(100)$ interface, we propose a method of precise interpretation of the evolution of the Auger signals recorded. Based on simple models of In crystallites, the AES intensities are calculated and compared with experimental results obtained. 


\section{Results}

It has been observed $[1,3]$ that under the $\mathrm{Ar}^{+}$bombardment the ratio of the Auger intensities between $P$ and In signals remains nearly constant $(\approx 0.28)$. In this case only some kinds of indium crystallites can be formed on the phosphorus rich surface.

The crystallites can be described in a simple way as square stacks of $h$ indium monolayers. Let us denote by $\mu$ the fraction of the surface covered by such In stacks. It is seen from Fig. 1 that only some pairs $(h, \mu)$ were observed. For most

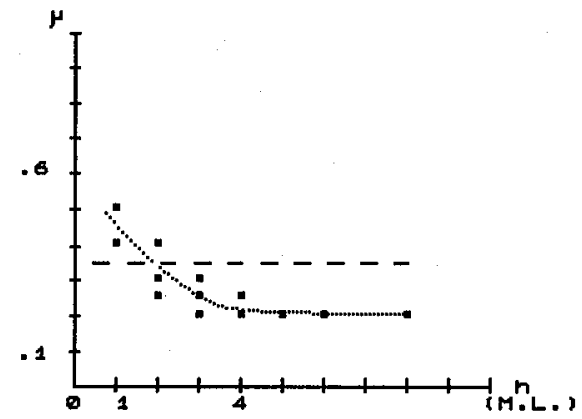

Fig. 1. Coverage $\mu$ of the surface as a function of the stack of $h$ indium monolayers (ML) for some possible pairs $(h, \mu)$.

of the values of $h$ such defined coverage $\mu$ is smaller than one half of the surface area uncovered by In crystallites. This implies: $\mu<(1-\mu) / 2$ or $\mu \leq 0.35$. It is also seen in Fig. 1 that the whole amount of In atoms present on the surface, which is proportional to $n=h \mu$, increases with increasing $h$ as the bombardment proceeds. The crystallites become greater while the value of $\mu$ is decreasing. On this kind of perturbed surfaces antimony deposits are formed already after short periods of exposure to the evaporation source $(t<15 \mathrm{~s})$. The determination of the absolute coverage was made using the deposition of the material onto a clean indium sample. One observes a Stranski-Krastanov [4] growth mode, one monolayer of Sb $\left(6 \times 10^{14}\right.$ atoms $/ \mathrm{cm}^{2}$ ) is condensed when the linear parts of the AES lines reach the ends.

The $\mathrm{Sb} / \mathrm{InP}(100)$ system is characterized by the transformations of the sample surface during the first stages of the deposition and the InSb layer formation. In such a case AES can be a useful method of analysis. However, for this kind of interface formation there is not a general formulation of the Auger intensities. The calculations can be made only if the disappearance of the crystallites and the states of the surface have been described.

\subsection{Calculation of the Auger current during the formation of the $S b / I n P$}

Before the cleaning treatment the surface of InP is recovered with a contamination layer consisting mainly of carbon. Using a low-energy $\left(300 \mathrm{eV}, 2 \mu \mathrm{A} / \mathrm{cm}^{2}\right)$ beam of $\mathrm{Ar}^{+}$ions, one monolayer of carbon can be removed in about $5 \mathrm{~min}$ [1]. After the clean substrate has been obtained a little prolonged $\mathrm{Ar}^{+}$treatment $\approx 5$ 
min) can induce generally one of the two following kinds of $3 \mathrm{D}$ indium clusters: $h=3 ; \mu=0.23$ or $h=4 ; \mu=0.25[3,6]$. In Fig. 2 the steps of the surface
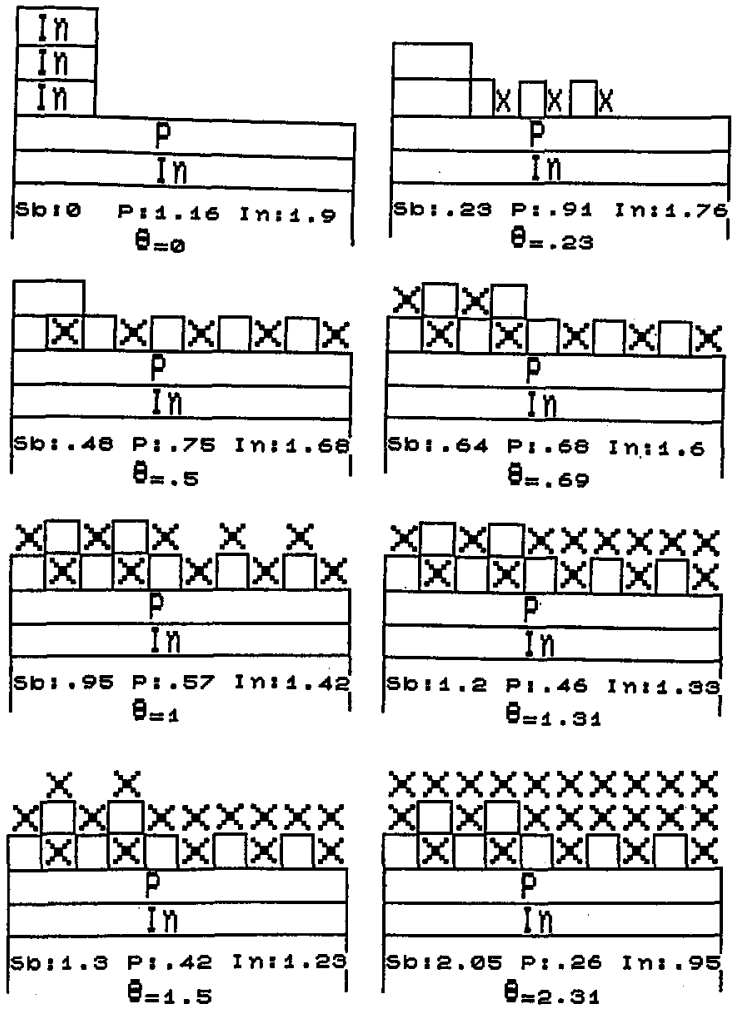

Fig. 2. The steps of the surface rearrangement during Sb deposition (initial state $h=3$ and $\mu=0.23$ ).

rearrangement for $h=3$ and $\mu=0.23$ are shown. The $\mathrm{Sb}$ and In atoms are bound, indium clusters are disappearing during the deposition. For each $\theta$ coverage of antimony it is possible to write the analytical expression of the AES intensities, taking into account the configuration of the sample surfaces. The $\mathrm{Sb}, \mathrm{P}$ and In AES signals are obtained knowing the attenuation coefficients of the Auger currents by an atomic monolayer: $\alpha_{\mathrm{Sb}}=0.74, \alpha_{\mathrm{P}}=0.56, \alpha_{\mathrm{In}}=0.72$ [7]. These signals are proportional to the numbers indicated in Fig. 2.

In the following we will explain in detail the calculation corresponding to the initial state of $\operatorname{InP}(100)$ and to the one after the deposition of one $\mathrm{Sb}$ monolayer. Each signal should be treated as a sum of distinct contributions because of the anisotropy of the surface. They originate from different parts of the sample and are represented in percentage by the numbers in the expressions given below. For $\theta=0$ (see Fig. 2) we have $I_{\mathrm{Sb}}=0$ and

$$
I_{\text {In }} \sim 0.77 \alpha_{\text {In }} /\left(1-\alpha_{\text {In }}^{2}\right)+0.23 \alpha_{\text {In }}^{4} /\left(1-\alpha_{\text {In }}^{2}\right)+0.23\left(1-\alpha_{\text {In }}^{3}\right) /\left(1-\alpha_{\text {In }}\right) \text {, }
$$


where the first, second and third term on the right hand side represent, respectively, the signal from the free surface $(1-\mu=0.77)$, the substrate through the crystallites $(\mu=0.23)$, the crystallites.

$$
I_{\mathrm{P}} \sim 0.77 /\left(1-\alpha_{\mathrm{P}}^{2}\right)+0.23 \alpha_{\mathrm{P}}^{3} /\left(1-\alpha_{\mathrm{P}}^{2}\right),
$$

where the first term on the right hand side represents the signal from the non-covered part of the surface, and the second one the signal through the crystallites.

For $\theta=1$ (Fig. 2) we have obtained a monolayer of an InSb compound on the substrate and

$$
I_{\mathrm{Sb}} \sim 0.81+0.19 \alpha_{\mathrm{Sb}}
$$

where 0.81 is the signal from the free $\mathrm{Sb}$ atoms on the surface (1st and 2nd layers) and $0.19 \alpha_{\mathrm{Sb}}$ is the signal from the $\mathrm{Sb}$ atoms of the $2 \mathrm{nd}$ layer covered by $\mathrm{In}$.

$$
I_{\text {In }} \sim 0.19+0.5 \alpha_{\text {In }}+0.69 \alpha_{\text {In }}^{3} /\left(1-\alpha_{\text {In }}^{2}\right)+0.31 \alpha_{\text {In }}^{2} /\left(1-\alpha_{\text {In }}^{2}\right) \text {. }
$$

Here, 0.19 is the signal from the In atoms in the first layer, $0.5 \alpha_{\text {In }}$ is the signal of the In atoms of the second monolayer. The third and fourth terms on the right hand side denote the signal from the substrate covered by three layers ( $\mathrm{Sb}$ or In and $\mathrm{P}$ ) and from the substrate covered by two monolayers (Sb and $\mathrm{P}$ ), respectively.

$$
I_{\mathrm{P}} \sim 0.69 \alpha_{\mathrm{P}}^{2} /\left(1-\alpha_{\mathrm{P}}^{2}\right)+0.31 \alpha_{\mathrm{P}} /\left(1-\alpha_{\mathrm{P}}^{2}\right) .
$$

The two terms on the right hand side represent the signal of the part of the substrate covered by two monolayers ( $\mathrm{Sb}$ and $\mathrm{In}$ ) and the signal of the substrate through the surface covered by only $\mathrm{Sb}$ atoms.
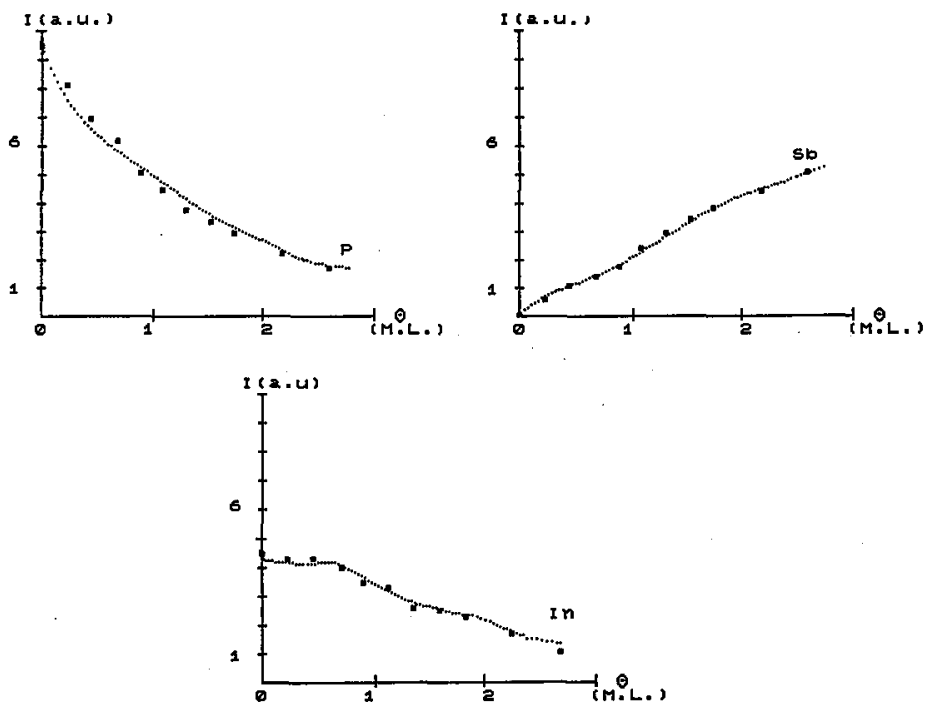

Fig. 3. The Auger amplitudes of P-LMM, Sb-MNN and In-MNN versus antimony coverage (squares - experimental points, dotted lines - theoretical calculations). 
Calculations can be prolonged until $\theta>2 \mathrm{ML}$ is reached and we obtain the theoretical Auger lines (Fig. 3). A good agreement with the experimental results is observed.

This result induces the reliability of the proposed scheme for the $\mathrm{Sb} / \operatorname{InP}(100)$ formation. In our opinion, there is an interesting possibility to follow by AES the first stages of the adsorption accompanied by surface submicroscopic transformations. This growth mode is not a classical one in comparison with those known for the metal-metal system [4]. However, after the complete formation of $\mathrm{InSb}$, antimony growth follows roughly the layer-by-layer mechanism and after 6 ML only the Sb signal will be detected in AES spectra as deduced from Fig. 3.

Some complementary results, obtained by using EELS, are displayed in Fig. 4. At a coverage near $\theta=1.2$ we observe the total disappearance of the original

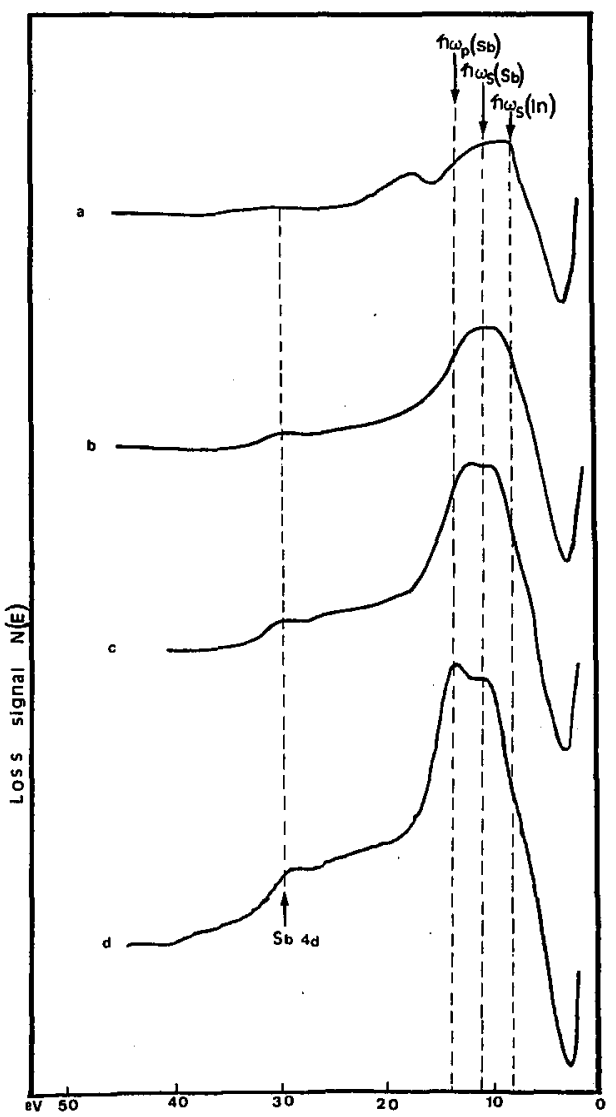

Fig. 4. Structure of the loss spectra recorded at a primary electron beam energy $E_{\mathrm{P}}=$ $500 \mathrm{eV}:$ (a) InP(100) just cleaned with In droplets, (b) after $\theta=1.2 \mathrm{ML}$, (c) $\theta=1.8 \mathrm{ML}$ of $\mathrm{Sb}$ deposit on the substrate, and (d) pure $\mathrm{Sb}$.

metallic indium structures (plasmon peaks $\hbar \omega_{p}, \hbar \omega_{s}$ ) and already the apparition 
of those of Sb. So this implies the end of the transformation process of the indium crystallites and the beginning of the formation of a pure antimony layer.

\subsection{The just cleaned bombarded surface}

In the previous paper [8] the authors have already mentioned that the just cleaned $\operatorname{InP}(100)$ corresponds to the following stacking arrangements: $3 / 4$ In + $1 / 4 \mathrm{P}, \mathrm{P}, \mathrm{In}, \mathrm{P}, \mathrm{In}, \ldots$ If the ionic beam is not stopped at time, the composition of the last layer can be changed and a small quantity of indium remains on the surface but yet no crystallites are formed (only perhaps some 2D islands). Using
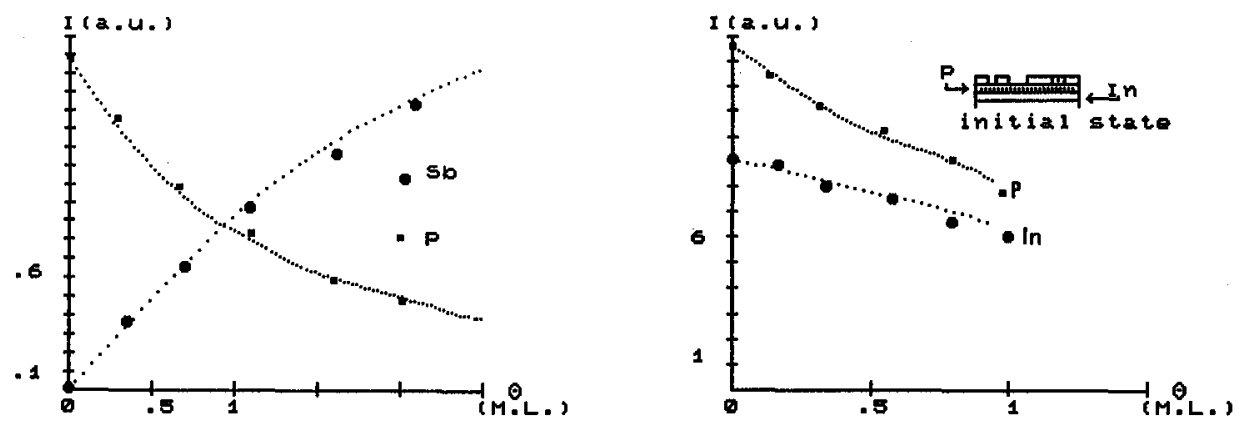

Fig. 5. Experimental points and theoretical dotted plots for the values of $\mathbf{P}$ and $\mathrm{Sb}$ Auger currents vs. $\mathrm{Sb}$ deposition.

Fig. 6. The variation of the Auger intensities for $\mathrm{P}$ and In vs. Sb deposition (dotted lines - calculated results).

AES results under Sb deposition (Fig. 5) we have obtained for the initial coverage of the P rich substrate by the In atoms: the case for which $\mu=0.4$. Intermediate cases corresponding to an incomplete change of the surface are possible, a part of the $\operatorname{In}_{0.75} \mathrm{P}_{0.25}$ two-dimensional compound is always present on the substrate. The Auger signal of phosphorus remains high during the $\mathrm{Sb}$ deposition: $\mathrm{P}$ atoms are carried up on the surface. Such a case is represented in Fig. 6. In this case we observe a good correlation between the calculated and recorded AES signals.

\subsection{Highly bombarded substrates}

Thick InSb layers on $\operatorname{InP}(100)$ samples can have attractive applications to further experiments (passivation of the surface, buffer layer for metal deposition on the semiconductor etc.). As they are directly related to the quantity of metallic indium present on the initial $\operatorname{In} P(100)$ surface, we should be interested in the results obtained from hard bombarded samples. Considering only the Auger signals of the $\mathrm{P}$ and $\mathrm{Sb}$ species, we obtain only a rough fitting of curves (Fig. 7). Moreover, a large difference is marked between the calculated and the experimental results for indium (Fig. 8). This discrepancy is increasing during the $\mathrm{Ar}^{+}$bombardment, and the evolution of the theoretical AES line is quite different to the recorded one. 


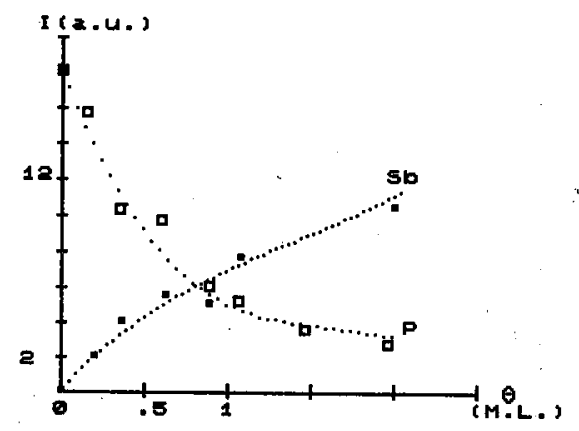

Fig. 7. $\mathrm{Pb}$ and $\mathrm{Sb}$ Auger signal intensities (dotted lines for the calculated ones) from a hard, initially perturbed surface vs. antimony evaporation.
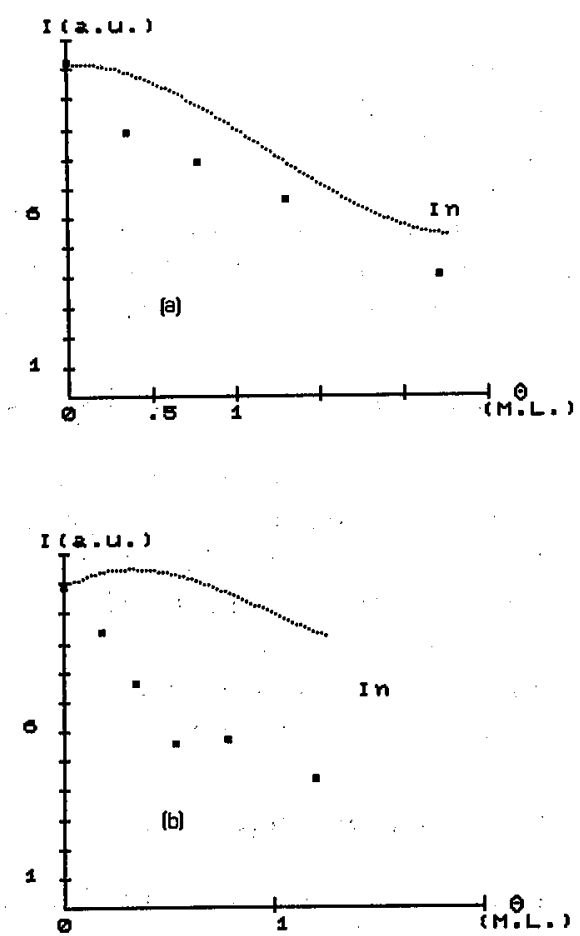

Fig. 8. Experimental (squares) and calculated (dotted lines) Auger intensities for indium vs. Sb coverage and with increasing time of $\mathrm{Ar}^{+}$bombardment: $(\mathrm{a}) \approx 1$ hour, (b) $\approx 2$ hours.

In fact, after a prolonged time of $\mathrm{Ar}^{+}$bombardment, indium crystallites form pyramids [9]. For this case in the model adopted above one can consider this shape of In crystallites at the initial step and for the first stages of the $\mathrm{Sb}$ condensation. 


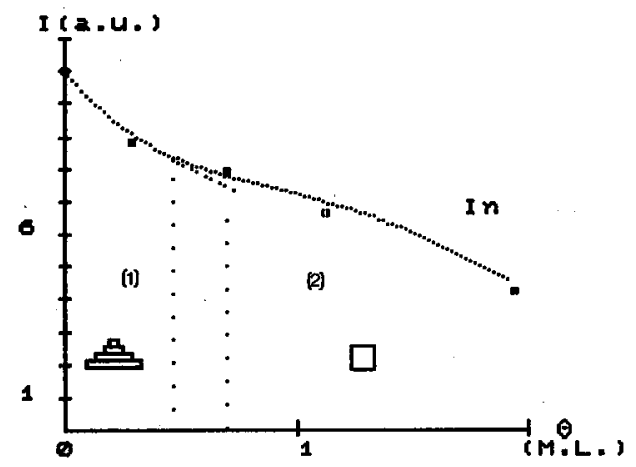

Fig. 9. Comparison of the calculated Auger intensities (dotted lines) with the experimental results using two different models (1 and 2) of In crystallites and their evolution during the $\mathrm{Sb}$ condensation.

The simple cubic model can be again considered when the In droplets are reduced until the end of the rearrangement process. By considering stepped shapes for the pyramidal In crystallites, each Auger current can be again calculated in a similar manner as described in Section 2.1 .

An example of the agreement between the calculated and experimental results of the In Auger signals is presented in Fig. 9. Two different regions (1 and 2) are corresponding to the two different forms considered for the indium crystallites.

\section{Summarizing remarks}

The AES and EELS techniques have been applied to investigate the steps of the $\mathrm{Sb} / \mathrm{InP}(100)$ interface formation. Using a simple model the experimental results are compared with the calculated ones. The good agreement obtained allows us to follow the microscopic variations of the structure and composition of the surface during the condensation of antimony.

The formation of the InSb layer can be controlled, and it brings about a great stability of the reordered surface and prevents interface migration. [10].

\section{Acknowledgements}

The authors would like to thank A. Mammar el Iladj for his contribution to some experimental AES results.

\section{References}

[1] B. Achard, B. Gruzza, C. Pariset, Surf. Sci. 160, L519 (1985).

[2] C. Jardin, D. Robert, B. Achard, B. Gruzza, C. Pariset, Surf. Interface Anal. 10, 301 (1987).

[3] B. Gruzza, C. Pariset, Surf. Sci. 247, 408 (1991). 
[4] J.A. Venables, G.D.T. Spiller, N. Hanbucken, Rep. Prog. Phys. 47, 399 (1984).

[5] S. Abdellaoui, B. Gruzza, C. Pariset, M. Bouslama, C. Jardin, D. Robert, Surf. Sci. 208, L21 (1989).

[6] B. Gruzza, Doctorat d'Etat, Blaise Pascal University Clermont-Ferrand, France, No. 389 (1987).

[7] B. Gruzza, C. Pariset, S. Abdellaoui, Surf. Interface Anal. 16, 54 (1990).

[8] B. Gruzza, B. Achard, C. Pariset, Surf. Sci. 162, 202 (1985).

[9] O. Wada, J. Phys. D, Appl. Phys. 17, 2429 (1984).

[10] S. Abdellaoui, Doctorat, Blaise Pascal University Clermont-Ferrand, France, No. 179 (1989). 